\title{
FROM BOLTZMANN EQUATION TO SPHERICAL HARMONICS EXPANSION MODEL: DIFFUSION LIMIT AND POISSON COUPLING*
}

\author{
MOHAMED LAZHAR TAYEB ${ }^{\dagger}$ \\ In the memory of Naoufel Ben Abdallah
}

\begin{abstract}
The diffusion approximation of an initial-boundary value problem for a BoltzmannPoisson system is studied. An elastic operator modeling electron-impurity collision is considered. A relative entropy is used to control the terms coming from the boundary and to prove useful $\mathrm{L}^{2}$-estimates for the renormalized solutions of the scaled Boltzmann equation (coupled to Poisson). A careful analysis of a relative entropy for high velocity allows us to show uniform bounds for the total mass and the kinetic energy which gives the compactness of the self-consistent electrostatic potential. Then, the moment method is used to prove the convergence of the renormalized solutions to a weak solution of a Spherical Harmonics Expansion (or SHE-) model coupled to the Poisson equation.
\end{abstract}

Key words. Kinetic transport equations, Boltzmann-Poisson system, Spherical Harmonics Expansion (SHE) model, relative entropy, electron-impurity collision, Diffusion limit, Macroscopic limit, Hilbert expansion.

AMS subject classifications. 35B45, 35B25, 82B21, 82B40, 54C70.

\section{Introduction}

In this paper, we study the diffusion approximation of a Boltzmann-Poisson system modeling electron-impurity interactions. We consider electrons described by their distribution function $f^{\alpha}:=f^{\alpha}(t, x, v)$ defined on the phase space $\Omega=\omega \times \mathbb{R}^{N},(N \geq 2)$. The time variable $t$ is nonnegative. The position $x$ belongs to a bounded and regular domain $\omega$. The velocity $v$ belongs to $\mathbb{R}^{N}$ and $\alpha$ is a nonnegative parameter on the order of the mean free path of collisions. These particles are submitted to a selfconsistent electric potential $\phi^{\alpha}(t, x)$ created by the electrons themselves and a given stationary external potential $\tilde{\phi}_{b}(x)$. The scaled Boltzmann equation reads as follows:

$$
\frac{\partial f^{\alpha}}{\partial t}+\frac{1}{\alpha}\left(v \cdot \nabla_{x} f^{\alpha}-\nabla_{x}\left(\phi^{\alpha}+\tilde{\phi}_{b}\right) \cdot \nabla_{v} f^{\alpha}\right)-\frac{Q\left(f^{\alpha}\right)}{\alpha^{2}}=0 .
$$

The initial data is a given function

$$
f^{\alpha}(0, x, v)=f_{0},
$$

and the inflow boundary data is well prepared; it is a function $\gamma$ depending on $(x, v)$ via the total energy $\left(|v|^{2} / 2+\phi_{b}(x)\right)$ :

$$
f^{\alpha}(t, x, v)=f_{b}(t, x, v):=\gamma\left(\frac{1}{2}|v|^{2}+\phi_{b}(x)\right), \quad v \cdot n(x)<0, x \in \partial \omega
$$

where $n(x)$ is the outward unit normal vector at the position $x$ on the boundary $\partial \omega$.

\footnotetext{
${ }^{*}$ Received: February 13, 2009; accepted (in revised version): July 25, 2010. Communicated by Francois Golse.

${ }^{\dagger}$ Department of Mathematics, University of Tunis ElManar, Faculty of Sciences of Tunis, (lazhar.tayeb@fst.rnu.tn).
} 
The elastic operator modeling electron-impurity interactions is given by

$$
Q(f)(v)=\int_{\mathbb{R}^{N}} s\left(x, v, v^{\prime}\right) \delta\left(\left|v^{\prime}\right|^{2}-|v|^{2}\right)\left(f\left(v^{\prime}\right)-f(v)\right) d v^{\prime},
$$

where, $s\left(x, v, v^{\prime}\right) d v^{\prime} d t$ is the probability density for a particle having a velocity $v$ to exit the interaction zone, with a new velocity $v^{\prime}$ belonging to the volume $d v^{\prime}$ around $v^{\prime}$, and during a time interval $d t$, and $\delta$ is a Dirac delta measure.

Let $v=\sqrt{2 \varepsilon}, \varepsilon=|v|^{2} / 2$, and $w \in v /|v| \in S^{N-1}$ where $S^{N-1}$ is the unit sphere of $\mathbb{R}^{N}$. By identifying $v$ with $(\varepsilon, w)$ one can write the co-area formula, for all integrable function $\varphi$ on $\mathbb{R}^{N}$, as

$$
\int_{\mathbb{R}^{N}} \varphi(v) d v=\int_{\varepsilon>0}(2 \varepsilon)^{N / 2-1} \int_{w \in S^{N-1}} \varphi(\varepsilon, w) d w d \varepsilon
$$

It follows, according to this formula, that

$$
Q(f)(v)=\int_{S^{N-1}}|v|^{N-2} \sigma(x, v,|v| w)[f(|v| w)-f(v)] d w .
$$

The potential $\phi^{\alpha}$ is a self-consistent solution of the homogeneous Poisson equation

$$
-\Delta_{x} \phi^{\alpha}(t, x)=\rho^{\alpha}(t, x):=\int_{\mathbb{R}^{N}} f^{\alpha}(t, x, v) d v .
$$

The potential $\tilde{\phi}_{b}$ is the harmonic extension in $\bar{\omega}$ of a given boundary data $\phi_{b}$ assumed to be smooth and nonnegative.

In the present analysis, the solutions for the Boltzmann equation are considered in a renormalized sense. This concept will be formulated later on, in Definition 5.1.

Our aim is to prove the convergence of the renormalized solutions $\left(f^{\alpha}, \phi^{\alpha}\right)$ of the Boltzmann-Poisson system (1.1)-(1.5), as $\alpha$ goes to zero, towards a function $(F, \phi)$ satisfying a Spherical Harmonics Expansion (or SHE-)model coupled to the Poisson equation. We notice that the SHE-model is an initial-boundary value problem

$$
(\sqrt{2 \varepsilon})^{N-2} \frac{\partial F}{\partial t}-\left(\nabla_{x}-\nabla_{x}\left(\phi+\tilde{\phi}_{b}\right) \frac{\partial}{\partial \varepsilon}\right)\left[D(\varepsilon)\left(\nabla_{x} F-\nabla_{x}\left(\phi+\tilde{\phi}_{b}\right) \frac{\partial F}{\partial \varepsilon}\right)\right]=0,
$$

where the unknown $F=F(t, x, \varepsilon)$ is a distribution function depending on time, position, and kinetic energy. It is convenient to denote the "total" gradient $\tilde{\nabla}_{x, \varepsilon}$ by

$$
\tilde{\nabla}_{x, \varepsilon}=\nabla_{x}-\nabla_{x}\left(\phi+\tilde{\phi}_{b}\right) \frac{\partial}{\partial \varepsilon}
$$

and rewrite the SHE-Equation (1.6) as a spectral continuity equation

$$
(\sqrt{2 \varepsilon})^{N-2} \frac{\partial F}{\partial t}+\tilde{\nabla}_{x, \varepsilon} . J=0,
$$

where $J$ is a spectral current density

$$
J(t, x, \varepsilon)=-D(\varepsilon) \tilde{\nabla}_{x, \varepsilon} F,
$$


and $D(\varepsilon)$ is a diffusion matrix

$$
D(\varepsilon)=-(2 \varepsilon)^{N / 2} \int_{S^{N-1}} w \otimes Q^{-1}(w) d w .
$$

Initial and boundary conditions on $F$ are

$$
F(0, x, \varepsilon)=\frac{1}{\left|S^{N-1}\right|} \int_{S^{N-1}} f_{0}(x, \sqrt{2 \varepsilon} w) d w \quad \text { and } \quad F(t, x, \varepsilon)_{\mid x \in \partial \omega}=\gamma\left(\varepsilon+\tilde{\phi}_{b}(x)\right),
$$

where the function $\gamma$ is introduced in (1.3). Finally, the potential $\phi$ (appearing in (1.7)) solves the following homogeneous Poisson equation

$$
-\Delta_{x} \phi(t, x)=\left|S^{N-1}\right| \int_{0}^{+\infty} F(t, x, \varepsilon)(\sqrt{2 \varepsilon})^{N-2} d \varepsilon .
$$

Kinetic and fluid models have been, for many years, powerful tools to study transport phenomena in semiconductor physics [58,61, 66, 67, 69], plasma [41], gas dynamics [57, 37, 38], biology, and recently in traffic networks [51].

Fluid models like Drift-Diffusion, Energy-Transport and SHE equations, in semiconductor simulation, Keller-Segel modeling biological cell dynamics, and many others, are based on specific assumptions of the equilibrium regime. However, due to the ongoing miniaturization effort in nano-technology, systems of particles are generally far from equilibrium. Therefore, microscopic models are required for an accurate description of the motion. Kinetic models like Vlasov, Boltzmann, and Fokker-PlankLandau equations are recommended to describe systems in non-equilibrium state. In this family of micro-models, the evolution can be interpreted in terms of characteristics in position-velocity space. Kinetic formulation introduces the individual velocity as an additional variable to the position and time coordinates; it takes into account the interaction between particles by considering operators dealing with physical conservation properties of the dynamics.

In the literature of asymptotic analysis, many macroscopic models have been derived by assuming the smallness of the scaled mean free path of collisions (also called Knudsen number). The Diffusion limit $[20,26]$ and high-field approximations [13, $19,53,56]$ represent the art of such modelling techniques. Drift-Diffusion equations were introduced by Shockley in 1960 [17, 29]. In the 1990s, Poupaud rigorously derived a Drift-Diffusion equation from Boltzamnn statistics [55]. The analysis is based on a Hilbert expansion [37, 38]. In [32] a Drift-Diffusion equation for degenerate semiconductors is also obtained from Fermi-Dirac statistics using a velocity averaging lemma $[24,25,30,31,44,47]$. We notice that various techniques are developed to justify these asymptotics in several physical contexts. Further details can be found in $[8,28,32,3,4]$ and references therein.

These methods of approximation have been applied to different dynamics of collisions, leading to an hierarchy of fluid equations lying in the gap of the full Boltzmann equation and the Drift-Diffusion one. The SHE model was recently derived (formally) as a diffusion limit of the Boltzmann equation by considering elastic collisions. In this dynamics only mass and kinetic energy are conserved during shocks. It appears that functions depending on $v^{2} / 2$ are formal equilibrium states.

For transport kinetic equations, Bardos [2] has studied the existence of weak solutions using the characteristics method for a Vlasov transport equation with a given electrostatic field. Extensions of this method can be found in [56]. In [1, 12, 14, 
$5,52,60$ ] the existence of weak solutions for Vlasov-Poisson-Fokker-Planck systems is analyzed. The self variation of the electrostatic potential is also considered in several contexts. Solutions are constructed by using a compactness argument [40]. In [11], the concept of weak solution is extended to an initial boundary value problem for a one dimensional Boltzmann-Poisson system. In [22, 23], Diperna and Lions introduced the concept of renormalized solutions for the non linear Boltzmann equation. This concept of solution considers equations satisfied by some $\beta(f)$, and in many cases the mass conservation property and entropy are verified in a weak sense. This notion is then used by Mischler to construct a solution for the Boltzmann-Poisson system in a bounded domain $[49,50]$. Various details and eventually extensions are made by Le Bris and Lions [42], Bouchut [15] and also De-Lellis [21]. In [47], Masmoudi and Tayeb constructed a renormalized solution for an initial and inflow boundary value problem for the Boltzmann-Poisson system.

In addition to the non-linearity due to self consistency, it remains unclear how initial and boundary data should be considered to expect more regularity of the solutions. It seems that boundary considerations limit the regularity of the solutions and induce layers $[55,20]$. Many analyses are limited to well prepared initial and boundary data to avoid the high layers effect and the instability due to the entropy production terms. The asymptotic analysis for general inflow data is an interesting task.

The paper is organized as follows. In the next section, we present the assumptions considered throughout the analysis and we state the convergence result. In Section 3 , we recall the spectral properties of the collision operator. In Section 4, we derive formally the SHE model from the Boltzmann equation for a force-free case $\left(\phi^{\alpha}:=\phi\right)$. Section 5 is devoted to the existence of a renormalized solution for the BoltzmannPoisson system. In Section 6, we shall establish useful estimates for the solution of the scaled Boltzmann equation. In the spirit of [7], a relative entropy allows us to approximate the production terms due to the boundary for well prepared incoming boundary data. This strategy gives a uniform $\mathrm{L}^{2}$-estimate on the distribution function and controls the distance between $f^{\alpha}$ and its local equilibrium $F^{\alpha}$ only in $\mathrm{L}^{2}$. In Section 7 , we improve the obtained a priori estimates by proving that the total mass and the kinetic energy are uniformly bounded in $L^{1}$ (Lemma 7.1). Using this lemma, we obtain a (uniform) weak sense for the continuity equation and show the compactness for the electrostatic potential $\phi^{\alpha}$ in some $L^{p}$ to finish the proof of Theorem 2.1.

\section{Preliminaries and convergence result}

In this paragraph, we would like to precisely state the assumptions on the initial data, for the doping profile $\gamma$, and the dynamics of collisions. These properties are useful for constructing a renormalized solution for the Boltzmann-Poisson system and analyzing the diffusion limit.

2.1. Notations. The incoming and outgoing parts of the boundary, $\partial \Omega=$ $\partial \omega \times \mathbb{R}^{N}$, are denoted by

$$
\partial \Omega^{ \pm}=\{(x, v) \in \partial \Omega: \pm v \cdot n(x)>0\} .
$$


The spectral particles density $F^{\alpha}$ and the spectral current $J^{\alpha}$ are defined by

$$
\begin{aligned}
& F^{\alpha}(t, x, \varepsilon)=\frac{1}{\left|S^{N-1}\right|} \int_{S^{N-1}} f^{\alpha}(t, x, \sqrt{2 \varepsilon} w) d w \\
& J^{\alpha}(t, x, \varepsilon)=\frac{(\sqrt{2 \varepsilon})^{N-1}}{\alpha\left|S^{N-1}\right|} \int_{S^{N-1}} f^{\alpha}(t, x, \sqrt{2 \varepsilon} w) w d w .
\end{aligned}
$$

The total particles and current densities are defined by

$$
\begin{array}{ll}
\rho^{\alpha}(t, x):=\left|S^{N-1}\right| \int_{0}^{+\infty}(\sqrt{2 \varepsilon})^{N-2} F^{\alpha}(t, x, \varepsilon) d \varepsilon & =\int_{\mathbb{R}^{N}} f^{\alpha}(t, x, v) d v \\
j^{\alpha}(t, x):=\left|S^{N-1}\right| \int_{0}^{+\infty} J^{\alpha}(t, x, \varepsilon) d \varepsilon & =\frac{1}{\alpha} \int_{\mathbb{R}^{N}} f^{\alpha}(t, x, v) v d v .
\end{array}
$$

For any function $f \in L_{l o c}^{1}$, we denote by $\Phi[f]$ the unique self-consistent potential, i.e. the solution of the homogeneous Dirichlet problem

$$
-\Delta_{x} \Phi[f](x)=\int_{\mathbb{R}^{N}} f(x, v) d v, \quad x \in \omega .
$$

We define the functional $\beta_{\gamma}$ by

$$
\beta_{\gamma}(f)=-\int_{0}^{f} \gamma^{-1}(s) d s
$$

The relative entropy and the entropy production flux are given by

$$
\mathcal{R}_{\gamma}[f \mid g]=\int_{\Omega}\left(\beta_{\gamma}(f)-\beta_{\gamma}(g)-(f-g) \beta_{\gamma}^{\prime}(g)\right)+\frac{1}{2} \int_{\omega}|\nabla \Phi[f-g]|^{2} d x
$$

and

$$
\mathcal{R}_{\gamma}^{+}[f \mid g]=\int_{\partial \Omega^{+}}\left(\beta_{\gamma}(f)-\beta_{\gamma}(g)-(f-g) \beta_{\gamma}^{\prime}(g)\right)|v \cdot n(x)| d \sigma_{x} d v .
$$

As $\gamma$ is a decreasing function, this implies that $\beta_{\gamma}$ is strictly convex and that the relative entropy is nonnegative and vanishing only for $f=g$.

We define the kinetic equilibrium profile, or Gibbs state, as

$$
G(x, v)=\gamma\left(\frac{1}{2}|v|^{2}+\Phi[G](x)+\tilde{\phi}_{b}(x)\right) .
$$

We notice that this function plays a fundamental role in our analysis. We remark that it is stationary, belonging to the null space $\mathcal{N}(Q)$, and is constant over some characteristics lines:

$$
\frac{\partial G}{\partial t}=Q(G)=v \cdot \nabla_{x} G-\nabla_{x}\left[\Phi[G]+\tilde{\phi}_{b}\right] \cdot \nabla_{v} G=0 .
$$


2.2. Assumptions. From now on, we shall make the following assumptions (A1): (Initial state). The initial data $f_{0}$ is a nonnegative function satisfying

$$
f_{0} \in L_{x, v}^{\infty}, \quad \mathcal{R}_{\gamma}\left[f_{0} \mid G\right]<+\infty .
$$

(A2): (Micro-reversibility). The dynamics of collisions satisfies the detailed balance principle, in the sense that the cross section $\sigma$ is symmetric. We assume also that it is bounded from below and above:

$$
\forall(x, v, w) \in \omega \times \mathbb{R}^{N} \times S^{N-1}, \quad 0<\sigma_{1} \leq \sigma(x, v,|v| w)=\sigma(x,|v| w, v) \leq \sigma_{2} .
$$

(A3): The incoming profile $\gamma$ is defined on $\left[\inf _{x \in \partial \omega} \phi_{b},+\infty[\right.$ with values in $] 0,+\infty[$, smooth, strictly decreasing, and rapidly decreasing at infinity, so that $\gamma^{\prime}$ is bounded in $] 0, \infty[$ and

$$
\int_{0}^{+\infty} \gamma(s) s^{N / 2} d s<+\infty
$$

(A4): (Strict convexity at infinity).

$$
(\sqrt{u}-\sqrt{v})^{2} \lesssim\left(\gamma^{-1}(u)-\gamma^{-1}(v)\right)(v-u) \quad \text { for } u, v \in[0,1],
$$

where the notation $a \lesssim b$ means that $a=\mathcal{O}(b)$.

(A5) : There exist a constant $\eta>0$ and a function $\theta:[0, \eta] \rightarrow[0,1]$, satisfying

$$
\theta(s)>s, \quad \int_{0}^{+\infty} s^{N / 2-1} \theta(\gamma(s))<+\infty,
$$

and

$$
0 \leq \gamma^{-1}(s) \lesssim \gamma^{-1}(s)-\gamma^{-1}(\theta(s)) \quad \text { on }[0, \eta] \text { a.e. }
$$

\subsection{The convergence result.}

THEOREM 2.1. Assume that $(A 1)-(A 5)$ are satisfied. Let $\left(f^{\alpha}, \phi^{\alpha}\right)$ a renormalized solution of the scaled Boltzmann equation coupled to Poisson. Then, when $\alpha$ goes to zero,

$$
\begin{array}{ll}
f^{\alpha} \stackrel{*}{\rightarrow} F(t, x, \varepsilon) & \text { in } L^{\infty}\left(\mathbb{R}^{+} ; L^{\infty} \cap L^{1}\right), \\
\nabla_{x} \phi^{\alpha} \rightarrow \nabla_{x} \phi & \text { in } L^{2}\left(\mathbb{R}^{+} ; L^{p}\right), \quad \forall p \in[1,2[,
\end{array}
$$

where $(F, \phi)$ is a weak solution of the (SHE-P) model (1.8)-(1.12). Moreover,

$$
\phi \in L^{\infty}\left(\mathbb{R}^{+} ; W^{2, \frac{N+2}{N}} \cap H_{0}^{1}(\omega)\right)
$$

\section{Spectral properties of collisions}

Lemma 3.1. Assume that (A2) holds. Then,

1. $-Q$ is a bounded, symmetric and nonnegative operator on $L^{2}\left(\mathbb{R}^{N}, \psi\left(|v|^{2}\right) d v\right)$ for every positive weight function $\psi$. Moreover, it satisfies, for any measurable function $\psi \equiv \psi\left(|v|^{2}\right)$,

$$
Q(\psi f)=\psi Q(f)
$$


2. $\mathcal{N}(Q)=\left\{f: \exists g\right.$ such that $\left.f(v)=g\left(|v|^{2}\right)\right\}$.

3. $P_{\varepsilon} f(t, x, \varepsilon)=\frac{1}{\left|S^{N-1}\right|} \int_{S^{N-1}} f(t, x, \sqrt{2 \varepsilon} w) d w$ is the orthogonal projection of $f$ onto $\mathcal{N}(Q)$.

4. $\mathcal{R}(Q)=\mathcal{N}(Q)^{\perp}=\left\{f:=f(\varepsilon)\right.$ such that $\left.P_{\varepsilon} f=0, \quad \forall \varepsilon\right\}$.

5. For every $g \in \mathcal{R}(Q)$, the Equation $Q(f)=g$ has a unique solution $f \in \mathcal{R}(Q)$.

If $g$ is even (odd) then $f$ is even (odd).

6. $-Q$ is coercive on $\mathcal{R}(Q)$ :

$$
-\int_{S^{N-1}}[Q(f) f](\sqrt{2 \varepsilon} w) d w \geq \sigma_{1}(\sqrt{2 \varepsilon})^{N-2} \int_{S^{N-1}}\left[\left(f-P_{\varepsilon} f\right)(\sqrt{2 \varepsilon} w)\right]^{2} d w .
$$

The next lemma follows from $(A 2)-(A 5)$.

Lemma 3.2. Let $f, g \in L^{2} \cap L^{\infty}$. Then, the following inequalities hold:

1. $\|f-g\|_{L^{2}(\Omega)}^{2}+\left\|\nabla_{x}(\Phi[f-g])\right\|_{L^{2}(\omega)}^{2} \lesssim \mathcal{R}_{\gamma}[f \mid g]$

2. $\|f-g\|_{L^{2}\left(\partial \Omega^{+} ;|v \cdot n| d \sigma_{x} d v\right)}^{2} \lesssim \mathcal{R}_{\gamma}^{+}[f \mid g]$

3. $\int_{\mathbb{R}^{N}} Q(f) \gamma^{-1}(f) d v \lesssim-\int_{\mathbb{R}^{N}}|v|^{N-2}\left(\sqrt{f(v)}-\sqrt{P_{\left|v^{2}\right| / 2} f}\right)^{2} d v$,

where $\mathcal{R}_{\gamma}$ and $\mathcal{R}_{\gamma}^{+}$are defined in (2.5).

Proof. According to (A3), the function $\gamma$ satisfies $0 \leq-\gamma^{\prime} \leq C$ which implies $-\left(\gamma^{-1}\right)^{\prime} \geq \frac{1}{C}$. As a consequence,

$$
\beta_{\gamma}(f)-\beta_{\gamma}(g)-(f-g) \beta_{\gamma}^{\prime}(g)=-\frac{1}{2}(f-g)^{2}\left(\gamma^{-1}\right)^{\prime}(\xi) \geq \frac{1}{2 C}(f-g)^{2} .
$$

By Integrating this inequality on $\Omega$ (resp. $\partial \Omega$ ) with respect to $d x d v$ (resp. $|v \cdot n| d \sigma d v$ ), we get the first and second inequalities. For the third one, we multiply $Q(f)$ by $\gamma^{-1}(f)$ and integrate with respect to $d v$. Using the co-area formula, we can write

$$
\int_{\mathbb{R}^{N}}\left[Q(f) \gamma^{-1}(f)\right] d v=\int_{0}^{+\infty}(2 \varepsilon)^{N-2} \int_{\left(S^{N-1}\right)^{2}} \sigma\left(x, \xi^{\prime}, \xi\right)\left[f\left(\xi^{\prime}\right)-f(\xi)\right] \gamma^{-1}(f)\left(\xi^{\prime}\right) d w^{\prime} d w d \varepsilon
$$

where $\xi=\sqrt{2 \varepsilon} w$ and $\xi^{\prime}=\sqrt{2 \varepsilon} w$.

By applying the transformation $\left(w, w^{\prime}\right) \mapsto\left(w^{\prime}, w\right)$, and using the symmetry of $\sigma$, we infer

$$
\int_{\mathbb{R}^{N}}\left[Q(f) \gamma^{-1}(f)\right]=\frac{1}{2} \int_{0}^{+\infty}(2 \varepsilon)^{N-2} \int_{\left(S^{N-1}\right)^{2}} \sigma\left(f^{\prime}-f\right)\left(\gamma^{-1}(f)\left(\xi^{\prime}\right)-\gamma^{-1}(f)\left(\xi^{\prime}\right)\right) d w^{\prime} d w d \varepsilon,
$$

where $\sigma$ is taken at $\left(x, \xi, \xi^{\prime}\right), f=f(\xi)$, and $f^{\prime}=f\left(\xi^{\prime}\right)$. Using Assumption (A4) and the fact that $\sigma$ is bounded from above, we obtain

$$
\begin{aligned}
\int_{\mathbb{R}^{N}}\left[Q(f) \gamma^{-1}(f)\right] & \leq \frac{-\sigma_{1}}{2} \int_{0}^{+\infty}(2 \varepsilon)^{N-2} \int_{\left(S^{N-1}\right)^{2}}\left[\sqrt{f\left(\xi^{\prime}\right)}-\sqrt{f(\xi)}\right]^{2} d w^{\prime} d w d \varepsilon \\
& \leq \frac{-\sigma_{1}}{2} \int_{\mathbb{R}^{N}}|v|^{N-2} \int_{S^{N-1}}[\sqrt{f(v)}-\sqrt{f(|v| w)}]^{2} d w d v
\end{aligned}
$$


By applying the Jensen inequality with the measure $d w /\left|S^{N-1}\right|$, and the convex function $t \mapsto(c-\sqrt{t})^{2}$ we get

$$
\frac{1}{\left|S^{N-1}\right|} \int_{S^{N-1}}[\sqrt{f(v)}-\sqrt{f(|v| w)}]^{2} d w \geq\left(\sqrt{f(v)}-\sqrt{P_{|v|^{2} / 2} f}\right)^{2},
$$

which leads to

$$
\int_{\mathbb{R}^{N}}\left[Q(f) \gamma^{-1}(f)\right] \lesssim-\int_{\mathbb{R}^{N}}|v|^{N-2}\left(\sqrt{f(v)}-\sqrt{P_{|v|^{2} / 2} f}\right)^{2} d v
$$

\section{Formal Hilbert expansion}

Let us consider the force-free case $\left(\phi^{\alpha}:=\phi\right)$ and assume that the given electrostatic potential $\phi$ is smooth enough. Insert the following Hilbert expansion of $f^{\alpha}$ into the linear scaled Boltzmann equation associated with $\phi$ :

$$
f^{\alpha}=f_{0}+\alpha f_{1, \phi}+\alpha^{2} f_{2, \phi}+\ldots
$$

Identifying terms with same powers of $\alpha$, we get

$$
\begin{aligned}
& Q\left(f_{0}\right)=0 \\
& Q\left(f_{1, \phi}\right)=v \cdot \nabla_{x} f_{0}-\nabla_{x}\left(\phi+\tilde{\phi}_{b}\right) \cdot \nabla_{v} f_{0} \\
& Q\left(f_{2, \phi}\right)=\frac{\partial f_{0}}{\partial t}+v \cdot \nabla_{x} f_{1, \phi}-\nabla_{x}\left(\phi+\tilde{\phi}_{b}\right) \cdot \nabla_{v} f_{1, \phi} .
\end{aligned}
$$

According to Lemma 3.1, the first (formal) approximation of $f^{\alpha}$ is an energydepending function: There exists a function $F$ such that

$$
f_{0}(t, x, v)=F\left(t, x, \frac{|v|^{2}}{2}\right) .
$$

By replacing $f_{0}$ by $F$ in $(4.2)$ we obtain

$$
Q\left(f_{1, \phi}\right)=v \cdot\left[\nabla_{x} F-\nabla_{x}\left(\phi+\tilde{\phi}_{b}\right) \frac{\partial F}{\partial \varepsilon}\right] .
$$

The function $F$ is even, so the right hand side of the above equation is an odd function of $v$. Therefore, using Lemma 3.1 we need only solve $Q(\lambda)=v \in \mathcal{R}(Q)$. Denoting by $\tilde{\nabla}_{x, \varepsilon}$ the transport operator

$$
\tilde{\nabla}_{x, \varepsilon}=\nabla_{x}-\nabla_{x}\left(\phi+\tilde{\phi}_{b}\right) \frac{\partial}{\partial \varepsilon}
$$

and by $\lambda$ the pseudo-inverse (componentwise) of $v$ :

$$
\lambda(v)=Q^{-1}(v):=|v| Q^{-1}(v /|v|) .
$$

Then, one can remark that $\lambda$ is an odd function of $v$ and then

$$
f_{1, \phi}=\lambda(v) \cdot \tilde{\nabla}_{x, \varepsilon} F+\theta\left(t, x,|v|^{2}\right),
$$

where $\theta$ is an arbitrary (even) function of $\mathcal{N}(Q)$. To solve (4.3), we define the spectral current density as

$$
J(t, x, \varepsilon)=(\sqrt{2 \varepsilon})^{N-1} \int_{S^{N-1}} f_{1, \phi}(t, x, \sqrt{2 \varepsilon} w) w d w .
$$


The solvability condition of (4.3) is equivalent to the following SHE equation:

$$
(\sqrt{2 \varepsilon})^{N-2} \frac{\partial F}{\partial t}+\tilde{\nabla}_{x, \varepsilon} \cdot J=0 .
$$

By replacing $J$ and $f_{1, \phi}$ by their expressions, we get

$$
J(t, x, \varepsilon)=-D(\varepsilon) \tilde{\nabla}_{x, \varepsilon} F
$$

where $D(\varepsilon)$ is the diffusion coefficient

$$
D(\varepsilon)=-(2 \varepsilon)^{N / 2} \int_{S^{N-1}}\left(w \otimes Q^{-1}(w)\right) d w .
$$

Finally, using Lemma 3.1, we deduce that $D(\varepsilon)$ is positive definite.

\section{Existence of solution}

Definition 5.1. Fix $\alpha>0, f^{\alpha} \in L_{l o c}^{1}$ and $\phi^{\alpha} \in W_{\text {loc }}^{1,1}$. We say that $\left(f^{\alpha}, \phi^{\alpha}\right)$ is a renormalized solution of the Boltzmann-Poisson system (1.1)-(1.5) if the function $\beta_{\gamma}\left(f^{\alpha}\right)$ is a weak solution of

$$
\left\{\begin{array}{l}
\alpha \frac{\partial \beta_{\gamma}\left(f^{\alpha}\right)}{\partial t}+v \cdot \nabla_{x} \beta_{\gamma}\left(f^{\alpha}\right)-\nabla_{v} \cdot\left[\nabla_{x}\left(\phi^{\alpha}+\tilde{\phi}_{b}\right) \beta_{\gamma}\left(f^{\alpha}\right)\right]+\frac{Q\left(f^{\alpha}\right)}{\alpha} \gamma^{-1}\left(f^{\alpha}\right)=0, \\
\beta_{\gamma}\left(f^{\alpha}\right) \mid \partial \Omega^{-}=\beta_{\gamma}\left(f_{b}^{\alpha},\right) \\
\beta_{\gamma}\left(f^{\alpha}\right)(t=0)=\beta_{\gamma}\left(f_{0}\right),
\end{array}\right.
$$

and $\phi^{\alpha}$ satisfies the homogeneous Dirichlet problem in the distribution sense.

Definition 5.2. We say that $(F, \phi)$ is a weak solution of the SHE model coupled to Poisson Equation (1.8)-(1.12) if

$$
\begin{aligned}
& F \in L_{l o c}^{2}\left(\mathbb{R}^{+} ;\left(1+\varepsilon^{N / 2}\right) d x d \varepsilon\right) \cap L_{t, x, \varepsilon}^{\infty}, \\
& \tilde{\nabla}_{x, \varepsilon} F \in L_{l o c}^{2}\left(\varepsilon^{N / 2-1} d x d \varepsilon d t\right), \quad \phi \in L^{\infty}\left(0, T ; H^{2} \cap H_{0}^{1}(\omega)\right),
\end{aligned}
$$

and for all $\psi \in \mathcal{D}\left(\mathbb{R}_{t}^{+} \times \omega \times \mathbb{R}_{*}^{+}\right)$,

$$
\begin{gathered}
\int_{\omega \times \mathbb{R}_{\varepsilon}^{+}}(\sqrt{\varepsilon})^{N-2} \\
\left(F \frac{\partial \psi}{\partial t}\right)(t, x, \varepsilon) d x d \varepsilon+\left.\int_{\omega \times \mathbb{R}_{\varepsilon}^{+}}(\sqrt{\varepsilon})^{N-2} F_{0} \psi\right|_{t=0} d x d \varepsilon \\
=\int_{\mathbb{R}_{t}^{+} \times \omega \times \mathbb{R}_{\varepsilon}^{+}}\left[D(\varepsilon) \tilde{\nabla}_{x, \varepsilon} F . \tilde{\nabla}_{x, \varepsilon} \psi\right] d t d x d \varepsilon
\end{gathered}
$$

and $\phi$ satisfies the homogeneous Dirichlet problem.

Theorem 5.3. Assume that (A1)-(A5) hold. Then, the Boltzmann-Poisson system (1.1)-(1.5) has a renormalized solution in the sense of the Definition 5.1. Moreover, it satisfies

1. $L^{\infty}$-estimate:

$$
0 \leq f^{\alpha} \leq \max \left(\liminf \left\|f_{0}\right\|_{\left.L^{\infty}, \gamma\left(\inf _{\partial \omega} \phi_{b}\right)\right)}\right.
$$


2. Spectral conservation property:

$$
\frac{\partial F^{\alpha}}{\partial t}+\tilde{\nabla}_{x, \varepsilon} \cdot J^{\alpha}=0
$$

where $F^{\alpha}$ and $J^{\alpha}$ are given in (2.2) and $\tilde{\nabla}_{x, \varepsilon}=\nabla_{x}-\nabla_{x}\left(\phi^{\alpha}-\tilde{\phi}_{b}\right) \frac{\partial}{\partial \varepsilon}$.

3. Continuity equation:

$$
\frac{\partial \rho^{\alpha}}{\partial t}+\nabla_{x} \cdot j^{\alpha}=0
$$

4. Entropy inequality:

$$
\frac{d}{d t} \mathcal{R}_{\gamma}\left[f^{\alpha} \mid G\right]+\frac{1}{\alpha} \mathcal{R}_{\gamma}^{+}\left[f^{\alpha} \mid G\right] \leq \frac{1}{\alpha^{2}} \int_{\Omega} Q(f) \gamma^{-1}(f) d x d v .
$$

For the sake of clarity, the proof of the theorem will be postponed in Appendix B. We notice that the present asymptotic analysis also gives a rigorous proof of the existence of a solution for the SHE-Poisson system satisfying Definition 5.2. We refer to [39] and references therein for recent developments on the existence of weak solutions for the Spherical Harmonics Expansion model coupled to the Poisson equation.

\section{Uniform a priori estimates}

Lemma 6.1. Assume that the initial data $f_{0} \in L_{+}^{\infty}(\Omega)$ and $\mathcal{R}_{\gamma}\left[f_{0} \mid G\right]<\infty$. Then, $f^{\alpha}$ satisfies the $L^{\infty}$ - uniform bound (5.2) and the following estimates:

1. $L^{2}$-estimate:

$$
\begin{array}{r}
{\left[\left\|f^{\alpha}-G\right\|_{L^{2}(\Omega)}^{2}+\left\|\nabla_{x} \Phi\left[f^{\alpha}-G\right]\right\|_{L^{2}(\omega)}^{2}\right]_{0}^{t}+\frac{1}{\alpha} \int_{0}^{t} \int_{\partial \Omega^{+}}\left(f^{\alpha}-f_{b}\right)^{2}(v \cdot n)} \\
\quad+\frac{1}{\alpha^{2}} \int_{0}^{t} \int_{\Omega \times S^{N-1}}|v|^{N-2}\left(\sqrt{f^{\alpha}(v)}-\sqrt{F^{\alpha}\left(|v|^{2} / 2\right)}\right)^{2} d v d w d s \lesssim 1 .
\end{array}
$$

2. $L^{1}$-identity:

$$
\left\|f^{\alpha}(t)\right\|_{L^{1}}+\frac{1}{\alpha} \int_{0}^{t} \int_{\partial \Omega^{+}}\left(f^{\alpha}(v)-f^{\alpha}(-v)\right)|v \cdot n(x)| d \sigma_{x} d v d s=\left\|f_{0}\right\|_{L^{1}} .
$$

Proof. The $\mathrm{L}^{2}$-estimate follows immediately by analyzing (5.5) using the inequalities stated in Lemma 3.2. The $\mathrm{L}^{1}$-identity is obtained from the weak formulation of the continuity Equation (5.4).

As a consequence of this lemma, we can establish the following Lemma 6.2.

1. $f^{\alpha}$ is uniformly bounded in $L^{\infty}\left(\mathbb{R}^{+}, L_{x, v}^{2}\right) \cap L_{t, x, v}^{\infty}$ and $F^{\alpha}$ is uniformly 2. bounded in $L^{\infty}\left(\mathbb{R}^{+}, L^{2}\left(\left(1+\varepsilon^{N / 2-1}\right) d x d \varepsilon\right)\right) \cap L_{t, x, \varepsilon}^{\infty}$.

$$
f^{\alpha}(t, x, v)=F^{\alpha}\left(t, x, \frac{|v|^{2}}{2}\right)+\alpha g^{\alpha}(t, x, v),
$$

where $g^{\alpha}$ is uniformly bounded in $\left[L^{1} \cap L^{2}\right]\left(|v|^{N-2} d t d x d v\right)$ and $F^{\alpha}=$ $P_{|v|^{2} / 2} f^{\alpha}$. 
3. The spectral current density $J^{\alpha}$ is uniformly bounded in $L_{t, x, \varepsilon}^{1}$.

4. The electrostatic field $\nabla_{x} \phi^{\alpha}$ is uniformly bounded in $L^{\infty}\left(\mathbb{R}^{+}, L^{2}(\omega)\right)$.

Proof. $f^{\alpha}$ is uniformly bounded in $L^{\infty}$ by identity (5.2); it is also the same for $F^{\alpha}(\varepsilon):=\left|S^{N-1}\right|^{-1} \int_{S^{N-1}} f^{\alpha}(\sqrt{2 \varepsilon} w) d w$. The decay of the profile $\gamma$ given in Assumption (A3) implies that the Gibbs state $G$ belongs to $L^{\infty}\left(\mathbb{R}^{+}, L_{x, v}^{2}\right) \cap L_{t, x, v}^{\infty}$, and then the $\mathrm{L}^{2}$-estimate (6.1) leads to the uniform bound for $f^{\alpha}$ in $L^{\infty}\left(\mathbb{R}^{+}, L^{2}\right)$ :

$$
\begin{aligned}
\int_{x, \varepsilon} F^{\alpha}(t, x, \varepsilon)^{2}\left(1+\varepsilon^{N / 2-1}\right) & =\int_{x, \varepsilon}\left(1+\varepsilon^{N / 2-1}\right)\left[\left|S^{N-1}\right|^{-1} \int_{S^{N-1}} f^{\alpha}(\sqrt{2 \varepsilon} w) d w\right]^{2} \\
& \leq \int_{x, \varepsilon}\left(1+\varepsilon^{N / 2-1}\right)\left[\left|S^{N-1}\right|^{-1} \int_{S^{N-1}} f^{\alpha}(\sqrt{2 \varepsilon} w)^{2} d w\right] \\
& \lesssim \int_{x} \int_{\varepsilon \leq 1} f^{\alpha}(\sqrt{2 \varepsilon} w)^{2}+\int_{x} \int_{\varepsilon \geq 1} \varepsilon^{N / 2-1} \int_{S^{N-1}} f^{\alpha}(\sqrt{2 \varepsilon} w)^{2} \\
& \lesssim m e s(\omega)\left|S^{N-1}\right|\left\|f^{\alpha}(t)\right\|_{L^{\infty}}^{2}+2\left\|f^{\alpha}(t)\right\|_{L^{2}}^{2} \leq C
\end{aligned}
$$

The second point is also a consequence of the $\mathrm{L}^{\infty}$ bound and Inequality (6.1). Indeed, by writing

$$
g^{\alpha}=\frac{1}{\alpha}\left(\sqrt{f^{\alpha}}-\sqrt{F^{\alpha}\left(|v|^{2} / 2\right)}\right)\left(\sqrt{f^{\alpha}}+\sqrt{F^{\alpha}\left(|v|^{2} / 2\right)}\right),
$$

we infer that

$$
\left|g^{\alpha}\right|^{2} \leq \frac{C}{\alpha^{2}}\left(\sqrt{f^{\alpha}}-\sqrt{F^{\alpha}\left(|v|^{2} / 2\right)}\right)^{2}
$$

which gives the $\mathrm{L}^{2}$-bound. The $\mathrm{L}^{1}$-bound is a consequence of the Helder inequality. Moreover, this distance to the local equilibrium gives a $\mathrm{L}^{1}$-bound of $J^{\alpha}$ and $j^{\alpha}$. Finally the uniform bound for the electrostatic field is obtained from (6.1) thanks to the good profile of the Gibbs state.

REMARK 6.3. We note that, at this stage, the solution of the Boltzmann-Poisson system only has a uniform bound in $L^{\infty}\left(\mathbb{R}^{+}, L_{x}^{p}\right)$ for all $p \in[2, \infty]$. The analysis used here does not allow one to obtain directly a uniform bound on $\left\|\rho^{\alpha}\right\|_{L^{1}(\omega)}=\left\|f^{\alpha}\right\|_{L^{1}(\Omega)}$. The main difficulty in obtaining such an estimate is approximating entropy dissipation terms for high velocities. In [47], the solution of the Boltzmann-Poisson system $f^{\alpha}$ belongs to $L \log L$, with bounded kinetic energy, but no $L^{p}$ estimate has been established for $p>1$. Here, we need a uniform $\mathrm{L}^{1}$-norm for the charge and current densities to prove compactness (in $t$ and $x$ ) for $\nabla_{x} \phi^{\alpha}$.

\section{7. $\mathbf{L}^{1}$-estimate}

Now, we would like to analyze the relative entropy dissipation $R_{\gamma}\left[f^{\alpha} \mid G\right]$ to improve the inequalities of Lemma 3.2 and give an $\mathrm{L}^{1}$ control on the distribution function $f^{\alpha}$.

Lemma 7.1. Under hypotheses $(A 1)-(A 5)$, the mass and the kinetic energy are uniformly bounded: $f^{\alpha}$ satisfies

$$
t \mapsto \int_{\Omega}\left(1+|v|^{2}\right) f^{\alpha}(t) d x d v \in L^{\infty}\left(\mathbb{R}^{+}\right) .
$$


Proof. The main idea to prove this lemma is to approximate the distribution function $f^{\alpha}$ for high velocities. Let us come back to the relative entropy $R_{\gamma}\left[f^{\alpha}(t) \mid G\right]$ and recall that $0 \leq R_{\gamma}\left[f^{\alpha}(t) \mid G\right]<+\infty$. More precisely, one can state

$$
\beta_{\gamma}\left(f^{\alpha}\right)-\beta_{\gamma}(G)-\left(f^{\alpha}-G\right) \beta_{\gamma}^{\prime}(G)=\int_{G}^{f^{\alpha}}\left(\gamma^{-1}(G)-\gamma^{-1}(s)\right) d s .
$$

By writing the density

$$
\rho^{\alpha}(t, x)=\int_{\left\{f^{\alpha} \leq \theta(G)\right\}} f^{\alpha} d v+\int_{\left\{f^{\alpha} \geq \theta(G)\right\}} f^{\alpha} d v,
$$

and using $(A 3)$, it is easy to see that

$$
\int_{\left\{f^{\alpha} \leq \theta(G)\right\}}\left(1+|v|^{2}\right) f^{\alpha} d v<+\infty .
$$

For the second integral, we divide the velocity space and write

$$
\int_{\left\{f^{\alpha} \geq \theta(G)\right\}} f^{\alpha} d v=\int_{\left\{|v| \leq A ; \theta(G) \leq f^{\alpha}\right\}} f^{\alpha} d v+\int_{\left\{|v| \geq A ; \theta(G) \leq f^{\alpha}\right\}} f^{\alpha} d v=I+I I .
$$

The first integral is finite using the fact that $f^{\alpha}$ is uniformly bounded in $L^{\infty}$. To approximate the second one, we choose $A \geq c$ such that for all $|v| \geq A$,

$$
\gamma\left(|v|^{2} / 2+\tilde{\phi}_{b}(x)+\Phi[G](x)\right)=G(x, v) \leq \frac{\eta}{2} \ll 1,
$$

and we remark that

$$
I I \leq\|G\|_{L^{1}}+\int_{\left\{|v| \geq A ; \theta(G) \leq f^{\alpha}\right\}}\left|f^{\alpha}-G\right| d v .
$$

The convexity of $\beta_{\gamma}$ gives

$$
\beta_{\gamma}\left(f^{\alpha}\right)-\beta_{\gamma}(G)-\left(f^{\alpha}-G\right) \beta_{\gamma}^{\prime}(G) \geq\left(\inf _{0 \leq s \leq 1}-\gamma^{-1^{\prime}}\left(G+s\left(f^{\alpha}-G\right)\right)\right)\left|f^{\alpha}-G\right|^{2} .
$$

The function $\gamma^{-1}$ is decreasing and $G \leq \theta(G) \leq f^{\alpha}$. This implies that

$$
-\gamma^{-1}\left(G+s\left(f^{\alpha}-G\right)\right) \geq-\gamma^{-1}\left(f^{\alpha}\right) \geq 0, \quad \forall s \in[0,1] .
$$

The assumption

$$
\exists a>0 \text { such that }-\gamma^{-1^{\prime}}\left(G+s\left(f^{\alpha}-G\right)\right) \geq a, \quad \forall s \in[0,1]
$$

implies that

$$
\int_{\left\{|v| \geq A ; \theta(G) \leq f^{\alpha} ;\left|f^{\alpha}-G\right| \geq \eta / 2\right\}}\left|f^{\alpha}-G\right| d v \leq \frac{2}{a \eta} R_{\gamma}\left[f^{\alpha}(t) \mid G\right]<+\infty .
$$


When $\left|f^{\alpha}-G\right| \leq \eta / 2$, we remark that $G \leq \theta(G) \leq f^{\alpha} \leq G+\eta / 2 \leq \eta$. We use the fact that the function $\beta_{\gamma}$ is increasing on $[0, \eta]$ to deduce

$$
\begin{aligned}
\beta_{\gamma}\left(f^{\alpha}\right)-\beta_{\gamma}(G)-\left(f^{\alpha}-G\right) \beta_{\gamma}^{\prime}(G) & =\left(|v|^{2} / 2+\tilde{\phi}_{b}+\Phi[G]\right)\left(f^{\alpha}-G\right)+\beta_{\gamma}\left(f^{\alpha}\right)-\beta_{\gamma}(G) \\
& \geq\left(|v|^{2} / 2+\tilde{\phi}_{b}+\Phi[G]\right)\left|f^{\alpha}-G\right|,
\end{aligned}
$$

and hence

$$
\int_{\left\{|v| \geq A ; \theta(G) \leq f^{\alpha} ;\left|f^{\alpha}-G\right| \leq \eta / 2\right\}}\left(|v|^{2} / 2+\tilde{\phi}_{b}+\Phi[G]\right)\left|f^{\alpha}-G\right| \leq \mathcal{R}_{\gamma}\left[f^{\alpha} \mid G\right]<+\infty .
$$

By combining (7.3) and (7.4) we control $f^{\alpha}$ for high velocities, so that the right hand side of (7.2) is finite. As a consequence, (7.1) is finite and the density $\rho^{\alpha}$ is uniformly bounded in $L^{1}(\omega)$. In the same way we show also that $f^{\alpha}$ is bounded in $L^{\infty}\left(\mathbb{R}^{+} ; L^{1}\left(|v|^{2} d x d v\right)\right)$. This completes the proof of Lemma 7.1.

\section{Passing to the limit}

Now we are able to conclude the proof of Theorem 2.1. We remark that the uniform bound of $f^{\alpha}$ in $L^{1}\left(1+|v|^{2}\right) \cap L^{\infty}$ implies that $\rho^{\alpha}$ is uniformly bounded in $L^{\infty}\left(\mathbb{R}^{+}, L^{\frac{N+2}{N}}\right)$. Using the Poisson equation, $\nabla_{x} \phi^{\alpha}$ is bounded in $L^{\infty}\left(\mathbb{R}^{+}, W^{1, \frac{N+2}{N}}\right)$. Moreover, using the entropy dissipation, we deduce that $j^{\alpha}$ is bounded in $L_{t, x}^{1}$ and then, from the continuity equation,

$$
\partial_{t} \rho^{\alpha}+\nabla_{x} \cdot j^{\alpha}=0
$$

we infer that $\partial_{t} \nabla_{x} \phi^{\alpha}$ is uniformly bounded in $L^{\infty}\left(\mathbb{R}^{+}, W^{-1,1}(\omega)\right)$. Now, by applying an Aubin lemma we get:

$$
\nabla_{x} \phi^{\alpha} \text { belongs to a compact subset of } L_{t, x}^{p}, \quad \forall p \in[1,2[\text {. }
$$

To finish the passage to limit $(\alpha \rightarrow 0)$ we need to identify the weak limit $J$ of the spectral current density $J^{\alpha}$.

Lemma 8.1. The current density $J$ satisfies

$$
J(t, x, \varepsilon)=-(2 \varepsilon)^{N / 2}\left[\int_{S^{N-1}}\left(w \otimes Q^{-1}(w)\right) d w\right] \tilde{\nabla}_{x, \varepsilon} F \in L_{t, x, \varepsilon}^{1} .
$$

where $\tilde{\nabla}=\nabla_{x}-\nabla_{x}\left(\phi^{\alpha}+\tilde{\phi}_{b}\right)$.

Proof. Using the relation

$$
f^{\alpha}=F^{\alpha}+\alpha g^{\alpha}
$$

and multiplying the scaled Boltzmann equation by $\alpha^{2}$, we get

$$
Q\left(g^{\alpha}\right)=\mathcal{O}(\alpha)_{\mathcal{D}^{\prime}}+v \cdot\left(\nabla_{x}-\nabla_{x}\left(\phi^{\alpha}+\tilde{\phi}_{b}\right) \nabla_{v}\right) F^{\alpha} .
$$

By passing to the limit, using the compactness of $\phi^{\alpha}$, we prove that there exist $F, \phi, g$ and $J$ satisfying

$$
\begin{array}{lll}
F^{\alpha} \quad \stackrel{*}{*} F, & \text { in } L^{1} \cap L^{\infty}, \\
\nabla_{x} \phi^{\alpha} \rightarrow \nabla_{x} \phi, & & \text { in } L_{t, x}^{p}, \\
g^{\alpha} \quad \rightarrow g, & & \text { in } L^{2}\left(|v|^{N-2} d t d x d v\right), \\
J^{\alpha} \quad \stackrel{*}{*} J, & \text { in } \mathcal{M}_{b} .
\end{array}
$$


In light of this convergence, one can infer from the above relation, using the fact that $F=F\left(|v|^{2} / 2\right)$ and $v \in \mathcal{R}(Q)$, that

$$
g=\left(\nabla_{x} F-\nabla_{x}\left(\phi+\tilde{\phi}_{b}\right) \frac{\partial F}{\partial \varepsilon}\right) Q^{-1}(v) .
$$

Furthermore, one can express $J$ as a function of $g$, so that

$$
\begin{aligned}
J: & =(\sqrt{2 \varepsilon})^{N-1} \int_{S^{N-1}} g w d w=(\sqrt{2 \varepsilon})^{N}\left[\int_{S^{N-1}} w \otimes Q^{-1}(w)\right]\left(\nabla_{x} F-\nabla_{x} \phi \frac{\partial F}{\partial \varepsilon}\right) \\
& =-D(\varepsilon)\left(\nabla_{x} F-\nabla_{x}\left(\phi+\tilde{\phi}_{b}\right) \frac{\partial F}{\partial \varepsilon}\right) \in L_{t, x, \varepsilon}^{1} .
\end{aligned}
$$

Now, we can pass to the limit (as $\alpha$ goes to zero) in the spectral equation satisfied by $F^{\alpha}$ and $J^{\alpha}$, thanks to the compactness of $\nabla_{x} \phi^{\alpha}$, and obtain

$$
\frac{\partial F}{\partial t}+\tilde{\nabla}_{x, \varepsilon} J=0, \quad \text { in } \mathcal{D}^{\prime}\left(\mathbb{R}_{t}^{+} \times \omega \times\right] 0, \infty[) .
$$

The $L^{2}$-estimate (6.1) gives the strong convergence of $\left(f^{\alpha}\right)_{\Gamma_{+}}$to the Gibbs state $G$. By passing to the limit in the Poisson equation we obtain that $(F, \phi)$ is a weak solution of the coupling SHE-Poisson system since $F$ and $J$ are in $\mathrm{L}^{1}$.

\section{Concluding remarks}

REMARK 9.1. We remark that the renormalized solutions for the Boltzamm-Poisson system have a weak sense. Indeed, the analysis of the entropy dissipation $\mathcal{R}\left[f^{\alpha} \mid G\right]$ allows us to prove, using interpolation arguments, that the charge and current densities belong to some $L^{p}$ for $p>1$. This is enough to give a weak sense for the renormalized solutions. We point out that in our analysis we need a uniform bound for the density and the kinetic energy in order to get a time-compactness for the potential.

REMARK 9.2. The analysis of diffusion approximation depends also on the regularity of the solution of the limit model. We remark that if the solution of the SHE-Poisson system has a Sobolev regularity, one can hope to obtain a rate of convergence using the Hybrid Hilbert expansion method. The additional difficulty in obtaining such a result is to prove that $\left(\alpha j^{\alpha}\right)$ converges to 0 in some $\mathrm{L}^{p}$ for $p>1$ (see $[11,48]$ for precise details). So, we can formally state

$$
\left\|\rho^{\alpha}-\rho_{F}\right\|_{L^{\infty}\left(0, T ; L^{1}(\omega)\right)} \lesssim \alpha^{1 / 3}
$$

Since this result is formal we restrict ourselves to present the 'approximation' of the current density. It is the subject of Appendix A.

\section{Appendix A. The current density.} Lemma A.1.

$$
\int_{0}^{\infty}\left\|j^{\alpha}(t)\right\|_{L^{\frac{6}{5}}} d t \lesssim \alpha^{-\frac{2}{3}}
$$

Proof. Let us write

$$
j^{\alpha}=\left|S^{N-1}\right| \int_{0}^{\infty} J^{\alpha}(\varepsilon) d \varepsilon=\int_{0}^{\infty} \frac{(\sqrt{2 \varepsilon})^{N-1}}{\alpha} \int_{S^{N-1}}\left(f^{\alpha}(\sqrt{2 \varepsilon} w)-F^{\alpha}(\varepsilon)\right) w d w d \varepsilon .
$$


Let $R>0$. Then,

$$
\begin{aligned}
\left|\alpha j^{\alpha}\right| \leq \int_{0}^{R^{2} / 2} & (\sqrt{2 \varepsilon})^{N-1} \int_{S^{N-1}}\left|\left(f^{\alpha}(\sqrt{2 \varepsilon} w)-F^{\alpha}\right)\right| d w d \varepsilon \\
& \quad+\int_{R^{2} / 2}^{\infty}(\sqrt{2 \varepsilon})^{N-1} \int_{S^{N-1}}\left|\left(f^{\alpha}(\sqrt{2 \varepsilon} w)-F^{\alpha}\right)\right| d w d \varepsilon:=I+I I .
\end{aligned}
$$

Using the $L^{\infty}$ bound of $f^{\alpha}$, we infer that

$$
\begin{aligned}
|I| & \leq \int_{0}^{R^{2} / 2} \int_{S^{N-1}}\left(f^{\alpha}-F^{\alpha}\right)(\sqrt{2 \varepsilon})^{N-1} d w d \varepsilon \\
& \leq\left(\left|S^{N-1}\right| \int_{0}^{R^{2} / 2}(2 \varepsilon) d \varepsilon\right)^{1 / 2}\left(\int_{0}^{\infty} \int_{S^{N-1}}\left(f^{\alpha}-F^{\alpha}\right)^{2}(2 \varepsilon)^{N-2} d w d \varepsilon\right)^{1 / 2} \\
& \lesssim \frac{R^{2}}{2}\left[\int_{0}^{\infty} \int_{S^{N-1}}\left(f^{\alpha}-F^{\alpha}\right)^{2}(2 \varepsilon)^{N-2} d w d \varepsilon\right]^{1 / 2}
\end{aligned}
$$

and

$$
|I I| \lesssim \frac{1}{R} \int_{0}^{\infty} \int_{S^{N-1}}(\sqrt{2 \varepsilon})^{N} F^{\alpha}(t, x, \varepsilon) d w d \varepsilon .
$$

Let $R$ be such that

$$
R^{3}=\frac{\int_{0}^{\infty} \int_{S^{N-1}}(\sqrt{2 \varepsilon})^{N} F^{\alpha}(t, x, \varepsilon) d w d \varepsilon}{\left[\int_{0}^{\infty} \int_{S^{N-1}}\left(f^{\alpha}-F^{\alpha}\right)^{2}(2 \varepsilon)^{N-2} d w d \varepsilon\right]^{1 / 2}}
$$

This choice of the radius $R$ is optimal and leads to

$$
\left|\alpha j^{\alpha}\right| \lesssim\left(\int_{0}^{\infty} \int_{S^{N-1}}(\sqrt{2 \varepsilon})^{N} F^{\alpha} d w d \varepsilon\right)^{2 / 3}\left[\int_{0}^{\infty} \int_{S^{N-1}}\left(f^{\alpha}-F^{\alpha}\right)^{2}(2 \varepsilon)^{N-2} d w d \varepsilon\right]^{1 / 6}
$$

or also, using the fact that the kinetic energy is bounded,

$$
\int_{0}^{T}\left\|j^{\alpha}(t)\right\|_{L^{6 / 5}} d t \leq \frac{C_{T}}{\alpha}\left[\int_{0}^{T} \int_{\omega} \int_{0}^{\infty} \int_{S^{N-1}}\left(f^{\alpha}-F^{\alpha}\right)^{2}(2 \varepsilon)^{N-2} d w d \varepsilon\right]^{1 / 6},
$$

so that

$$
\int_{0}^{T}\left\|j^{\alpha}(t)\right\|_{L^{6 / 5}} d t \leq \frac{C}{\alpha}\left[\int_{\omega} \int_{0}^{\infty} \int_{S^{N-1}}\left(f^{\alpha}-F^{\alpha}\right)^{2}(2 \varepsilon)^{N-2} d w d \varepsilon\right]^{1 / 6} \leq C \alpha^{-2 / 3}
$$

which ends the proof of (A.1).

Appendix B. Existence of a renormalized solution. Here we would like to prove the existence of a renormalized solution for the Boltzmann-Poisson system (1.1)-(1.5) (Theorem 5.2). The proof is divided in three steps, and to simplify the presentation we drop the parameter $\alpha$. In a first step, we regularize the BoltzmannPoisson system with a cut-off cross section and construct a uniformly bounded weak 
solution. Then, we remove the regularization of the Poisson operator. In a second step we establish a uniform entropy identity and finally we pass to the limit to obtain a renormalized solution for the unmodified system.

First step: Regularization. Let $R>0$. We define a cross section $\sigma_{R}$ :

$$
\sigma_{R}\left(x, v, v^{\prime}\right):=\sigma\left(x, v, v^{\prime}\right) 1_{|v| \leq R}(v) 1_{\left|v^{\prime}\right| \leq R}\left(v^{\prime}\right)
$$

and $Q_{R}$ :

$$
Q_{R}(f)(v):=|v|^{N-2} \int_{S^{N-1}} \sigma_{R}(x, v,|v| w)[f(|v| w)-f(v)] d w=Q_{R}^{+}(f)-\lambda_{R}(v) f(v),
$$

where $Q_{R}^{+}$stands for the gain part and $\lambda_{R}$ is an absorption term. The regularized Boltzmann-Poisson system is the following

$$
\left\{\begin{array}{lc}
\partial_{t} f+\left(v \cdot \nabla_{x} f-\nabla_{x}\left(\phi+\tilde{\phi}_{b}\right) \cdot \nabla_{v} f\right)=Q_{R}(f), & \\
-\left(1-\eta \Delta_{x}\right)^{2 m} \Delta_{x} \phi=\rho=\int_{\mathbb{R}^{N}} f d v, & \\
f(0, x, v)=f_{0}(x, v) \chi|v| \leq R, & (x, v) \in \partial \Omega^{-}, \\
f(t, x, v)=f_{b}=\gamma\left(|v|^{2} / 2+\phi_{b}\right), & x \in \partial \omega, \\
\phi=\Delta_{x} \phi=\ldots=\Delta_{x}^{2 m} \phi=0, &
\end{array}\right.
$$

where $\eta$ is a nonnegative parameter and $m \in \mathbb{N}^{*}$.

A standard computation shows that the operator $Q_{R}$ is bounded in $L^{2} \cap L^{\infty}$ and satisfies

$$
\int_{|v| \leq R} Q_{R}(f)\left(\begin{array}{l}
1 \\
|v|^{2}
\end{array}\right) d v=\int_{\mathbb{R}^{N}} Q_{R}(f)\left(\begin{array}{l}
1 \\
|v|^{2}
\end{array}\right) d v=\left(\begin{array}{l}
0 \\
0
\end{array}\right)
$$

It is also known that (B.3) is enough to apply the Schauder fixed-point procedure and construct a weak solution $\left(f_{\eta, R}, \phi_{\eta, R}\right)$ for (B.2). Moreover, the following assertions are satisfied:

- $f_{\eta, R}$ is uniformly bounded with respect to $\eta$ and $R$ in $L^{\infty} \cap L_{l o c}^{\infty}\left(\mathbb{R}^{+}, L_{x, v}^{1}\right)$.

- $f_{\eta, R}$ is uniformly bounded with respect to $\eta$ in $L_{l o c}^{\infty}\left(\mathbb{R}^{+}, L^{1}\left(|v|^{2} d v d x\right)\right)$.

- $\rho_{\eta, R}$ is uniformly bounded with respect to $\eta$ in $L_{l o c}^{\infty}\left(\mathbb{R}^{+}, L_{x}^{(N+2) / N}\right)$ and in $L_{l o c}^{\infty}\left(\mathbb{R}^{+}, L_{x, v}^{1}\right)$ with respect to $\eta$ and $R$.

- $j_{\eta, R}:=\int_{\mathbb{R}^{N}} v f_{\eta, R} d v$ is uniformly bounded in $L_{l o c}^{\infty}\left(\mathbb{R}^{+}, L_{x}^{(N+2) /(N+1)}\right)$ with respect to $\eta$.

- $\nabla_{x} \phi_{\eta, R}$ is relatively compact in $L_{l o c}^{2}\left(\mathbb{R}^{+}, L_{x}^{(N+2) /(N+1)}\right)$ uniformly with respect to $\eta$.

We refer to [11] for similar results. We remark that in present regularization we do not cut-off the velocity variable for the charge and current densities. We stress that we cut-off the initial data in order to assume only a finite relative entropy at $\mathrm{t}=0$. Generally, we assume that initial data should have a finite kinetic energy. 
By letting $\eta \rightarrow 0$, we obtain a weak solution $\left(f_{R}, \Phi\left[f_{R}\right], \rho_{R}, j_{R}\right)$ for

$$
(B P)_{R} \quad\left\{\begin{array}{l}
\partial_{t} f_{R}+v \cdot \nabla_{x} f_{R}-\nabla_{x}\left(\Phi\left[f_{R}\right]+\tilde{\phi}_{b}\right) \cdot \nabla_{v} f_{R}=Q_{R}\left(f_{R}\right), \\
f_{R}(0, x, v)=f_{0}(x, v) \chi_{|v| \leq R}, \\
f_{R}(t, x, v)=f_{b}=\gamma\left(|v|^{2} / 2+\phi_{b}\right), \quad(x, v) \in \partial \Omega^{-}, \\
-\Delta_{x} \Phi\left[f_{R}\right]=\rho_{R}=\int_{\mathbb{R}^{N}} f d v, \\
\Phi\left[f_{R}\right]=0, \quad x \in \partial \omega .
\end{array}\right.
$$

\section{Second step: Uniform entropy-dissipation}

LEMma B.1. Weak solutions of the modified system (B.4) satisfy the weak uniform maximum principle (5.2), the corresponding spectral and continuity equations stated in Theorem 5.3, and the following relative entropy:

$$
\frac{d}{d t} \mathcal{R}_{\gamma}\left[f_{R} \mid G\right]+\mathcal{R}_{\gamma}^{+}\left[f_{R} \mid G\right]+\int_{\Omega} Q_{R}\left(f_{R}\right) \gamma^{-1}\left(f_{R}\right)=0 .
$$

Proof. We begin by writing the Boltzmann equation as a Vlasov transport equation

$$
\partial_{t} f_{R}+v \cdot \nabla_{x} f_{R}-\nabla_{x}\left(\Phi\left[f_{R}\right]+\tilde{\phi}_{b}\right) \cdot \nabla_{v} f_{R}+\lambda_{R}(v) f_{R}=Q_{R}^{+}\left(f_{R}\right),
$$

noting that $\lambda_{R}$ is a nonnegative absorption term and $Q_{R}^{+}$preserves positivity. Using the characteristic method $[2,5]$ one can show that $f_{R}$ verifies the weak maximum principle (5.2). A suitable choice of a sequence of test functions allows to define the spectral Equation (5.3):

$$
\frac{\partial F_{R}}{\partial t}+\tilde{\nabla} \cdot J_{R}=0, \quad \text { in } \mathcal{D}^{\prime}\left(\mathbb{R}^{+} \times \bar{\omega} \times \mathbb{R}_{\varepsilon}^{+}\right),
$$

where the spectral density and current are

$$
F_{R}=\frac{1}{\left|S^{N-1}\right|} \int_{S^{N-1}} f_{R}(\sqrt{2 \varepsilon} w) d w, \quad J_{R}=\frac{(\sqrt{2 \varepsilon})^{N-1}}{\left|S^{N-1}\right|} \int_{S^{N-1}} f_{R}(\sqrt{2 \varepsilon} w) w d w
$$

and the total gradient is $\tilde{\nabla}:=\nabla_{x}-\nabla_{x}\left(\Phi\left[f_{R}\right]+\tilde{\phi}_{b}\right) \frac{\partial}{\partial \varepsilon}$.

In the same way we get a weak sense of the continuity Equation (5.4):

$$
\frac{\partial \rho_{R}}{\partial t}+\nabla_{x} \cdot j_{R}=0, \quad \text { in } \mathcal{D}^{\prime}\left(\mathbb{R}^{+} \times \bar{\omega}\right) .
$$

Furthermore, the function $\beta_{\gamma}\left(f_{R}\right)$ is a weak solution of (5.1) in the sense that we renormalize the initial and inflow boundary data:

$$
\beta_{\gamma}\left(f_{R}\right)_{\left.\right|_{\partial \Omega^{-}}}=\beta_{\gamma}\left(f_{\left.R\right|_{\partial \Omega^{-}}}\right) \quad \text { and } \quad \beta_{\gamma}\left(f_{R}(t=0)\right)=\beta_{\gamma}\left(f_{0} \chi_{|v| \leq R}\right) .
$$

Therefore,

$$
\frac{d}{d t} \int_{\Omega} \beta_{\gamma}\left(f_{R}\right)+\int_{\partial \Omega^{-} \cup \partial \Omega^{+}} \beta_{\gamma}\left(f_{R}\right)(v \cdot n(x))=\int_{\Omega} \beta_{\gamma}^{\prime}\left(f_{R}\right) Q_{R}\left(f_{R}\right) .
$$


Since the solution is weak and the initial data $\left.f_{R}\right|_{t=0} \in L^{1}\left(|v|^{2} d x d v\right)$, one can multiply the Boltzmann equation (satisfied by $f_{R}$ ) by $|v|^{2} / 2$ and get

$$
\begin{array}{r}
\frac{1}{2} \frac{d}{d t} \int_{\Omega}\left(|v|^{2}+\Phi\left[f_{R}\right]+2 \tilde{\phi}_{b}\right) f_{R}+\int_{\partial \Omega^{+}}\left(\frac{1}{2}|v|^{2}+\phi_{b}\right) f_{R}|v \cdot n(x)| \\
=\int_{\partial \Omega^{-}}\left(\frac{1}{2}|v|^{2}+\phi_{b}\right) f_{b}|v \cdot n(x)| .
\end{array}
$$

The continuity Equation (B.6) and the homogeneous Poisson equation $-\Delta_{x} \Phi\left[f_{R}\right]=\rho_{R}$ lead to

$$
\begin{gathered}
\frac{d}{d t}\left(\frac{1}{2} \int_{\Omega}\left(f_{R}-G\right) \Phi\left[f_{R}-G\right]\right)=\int_{\Omega} \frac{\partial}{\partial t}\left(f_{R}-G\right) \Phi\left[F_{R}-G\right] \\
=\int_{\Omega}\left(f_{R}-G\right) \frac{\partial}{\partial t} \Phi\left[f_{R}-G\right]=\frac{d}{2 d t}\left\|\nabla_{x} \Phi\left[f_{R}-G\right]\right\|_{L^{2}}^{2} .
\end{gathered}
$$

Moreover, $\Phi[G]$ vanishes on $\partial \omega$ and satisfies

$$
\frac{1}{2}|v|^{2}+\tilde{\phi}_{b}(x)=-\Phi[G]-\beta_{\gamma}^{\prime}(G), \quad \int_{\partial \Omega^{ \pm}}\left(\frac{1}{2}|v|^{2}+\phi_{b}\right) f_{R}=-\int_{\partial \Omega^{ \pm}} f_{R} \beta_{\gamma}^{\prime}(G),
$$

and by remarking that $\int \Phi[g] f=\int \Phi[f] g$, we can establish

$$
\frac{d}{2 d t} \int_{\Omega}\left[|v|^{2}+\Phi\left[f_{R}\right]+2 \tilde{\phi}_{b}\right] f_{R}=\frac{d}{d t} \int_{\Omega}\left[\frac{1}{2}\left(f_{R}-G\right) \Phi\left[f_{R}-G\right]-f_{R} \beta_{\gamma}^{\prime}(G)\right] .
$$

On the one hand, the sum of (B.7) and (B.8), according to the above identities, gives

$$
\begin{aligned}
\frac{d}{d t} \int_{\Omega}\left[\beta_{\gamma}\left(f_{R}\right)\right. & \left.+\frac{1}{2}\left(f_{R}-G\right) \Phi\left[f_{R}-G\right]-f_{R} \beta_{\gamma}^{\prime}(G)\right]+\int_{\Omega} Q_{R}\left(f_{R}\right) \gamma^{-1}\left(f_{R}\right) \\
& +\int_{\partial \Omega^{+}}|v \cdot n|\left(\beta_{\gamma}\left(f_{R}\right)-f^{\alpha} \beta_{\gamma}^{\prime}(G)\right)=\int_{\partial \Omega^{-}}|v \cdot n|\left(\beta_{\gamma}\left(f_{R}\right)-f_{R} \beta_{\gamma}^{\prime}(G)\right)
\end{aligned}
$$

On the other hand, the Gibbs state is even $(G(v)=G(-v))$ and does not depend on the time variable; this property leads to

$$
\frac{d}{d t} \int_{\Omega}\left(G \beta_{\gamma}^{\prime}(G)-\beta_{\gamma}(G)\right)=\sum_{ \pm} \pm \int_{\Gamma^{ \pm}}\left(G \beta_{\gamma}^{\prime}(G)-\beta_{\gamma}(G)\right)|v \cdot n|=0
$$

Finally, the sum of (B.9) and (B.10) gives (B.5) and ends the proof of Lemma B.1.

Third step: $(R \rightarrow 0)$.

As a consequence of Lemma 3.2, we deduce immediately that

- $f_{R}\left(\right.$ resp. $\left.F_{R}\right)$ is uniformly bounded in $L^{\infty}\left(\mathbb{R}^{+}, L_{x, v}^{2}\right)\left(\right.$ resp. $\left.L^{\infty}\left(\mathbb{R}^{+}, L_{x, \varepsilon}^{2}\right)\right)$.

- $J_{R}$ is uniformly bounded in $L_{l o c}^{1}\left(\mathbb{R}^{+}, L_{x, \varepsilon}^{1}\right)$.

- The trace $f_{\left.R\right|_{\partial \Omega^{+}}}$is uniformly bounded in $L^{\infty}\left(\mathbb{R}^{+}, L^{2}(|v \cdot n(x)| d x d v)\right)$.

- $\rho_{R}\left(\right.$ resp. $\left.j_{R}\right)$ is uniformly bounded in $L_{l o c}^{\infty}\left(\mathbb{R}^{+}, L^{1}\right)\left(\right.$ resp. $\left.L^{2}\left(\mathbb{R}^{+}, L^{1}\right)\right)$.

- $\nabla_{x} \Phi\left[f_{R}\right]$ is relatively compact in $L_{l o c}^{2}\left(\mathbb{R}^{+}, L_{x}^{p}\right)$ for all $p \in[1,2[$. 
With these estimates, we can pass to the limit $R \rightarrow 0$ and obtain the existence of $(f, \Phi[f])$ : a renormalized solution of the initial Boltzmann-Poisson system such that

$$
\begin{aligned}
& f_{R} \stackrel{L^{2}}{\rightarrow} f \\
& F_{R} \stackrel{L^{2}}{\rightarrow} F=\left|S^{N-1}\right|^{-1} \int_{S^{N-1}} f(\sqrt{2 \varepsilon}) d w, \\
& J_{R} \stackrel{\mathcal{M}}{b}^{b} J=\left|S^{N-1}\right|^{-1}(\sqrt{2 \varepsilon})^{N-1} \int_{S^{N-1}} f(\sqrt{2 \varepsilon}) w d w, \\
& \left(\rho_{R}, j_{R}\right) \stackrel{* \mathcal{M}}{b}^{b}(\rho, j)=\left(\int_{\mathbb{R}^{N}} f d v, \int_{\mathbb{R}^{N}} f v d v\right), \\
& -\Delta_{x} \Phi[f]=\rho, \quad \Phi[f]=0, \quad \text { on } \partial \omega .
\end{aligned}
$$

Moreover, the spectral equation and the continuity equation are satisfied in the weak sense, and by a convexity argument and a velocity averaging lemma we can state the relative entropy inequality:

$$
\mathcal{R}_{\gamma}[f \mid G](t)+\int_{0}^{t} \mathcal{R}_{\gamma}^{+}[f \mid G](s) d s+\int_{0}^{t} \int_{\Omega} Q(f) \gamma^{-1}(f) \leq \mathcal{R}_{\gamma}\left[f_{0} \mid G\right],
$$

which ends the proof of the existence of renormalized solution for Boltzmann-Poisson.

Acknowledgement. I would like to thank Nader Masmoudi for interesting discussions and the referee for constructive remarks.

\section{REFERENCES}

[1] R. Alexandre, Weak solutions of the Vlasov-Poisson initial-boundary value problem, Math. Meth. Appl. Sci., 16(8), 587-607, 1993.

[2] C. Bardos, Problèmes aux limites pour les équations aux dérivées partielles du premier ordre à coefficients réels; théorèmes d'approximation; application à l'équation de transport, Ann. Sci. de l'Ec. Norm. Sup., 4e série, 3, 185-233, 1970.

[3] C. Bardos, F. Golse and D. Levermore, Fluid dynamic limits of Kinetic equations. II: Convergence proofs for the Boltzmann equation, Commun. Pure Appl. Math., 46(5), 667-753, 1993.

[4] C. Bardos, F. Golse, B. Perthame and R. Sentis, The nonaccretive radiative transfer equations: existence of solutions and Rosseland approximation, J. Funct. Anal., 77(2), 434-460, 1988.

[5] N. Ben Abdallah, Weak solutions of the initial-boundary value problem for the Vlasov-Poisson system, Math. Meth. Appl. Sci., (17), 451-476, 1994.

[6] N. Ben Abdallah, H. Chaker and C. Schmeiser, The high field asymptotics for a fermionic Boltzmann equation: entropy solutions and kinetic shock profiles, J. Hyperbolic Diff. Eqs., 4, 679-704, 2007.

[7] N. Ben Abdallah and P. Degond, On a hierarchy of macroscopic models for semiconductors, J. Math. Phys., 37(7), 3306-3333, 1996.

[8] N. Ben Abdallah and J. Dolbeault, Relative entropies for kinetic equations in bounded domains (irreversibility, stationary solutions, uniqueness), Arch. Ration. Mech. Anal., 168(4), 253298, 2003.

[9] N. Ben Abdallah, L. Desvillettes and S. Génieys, On the convergence of the Boltzmann equation for semiconductors towards the energy transport model, J. Statist. Phys., 98(3-4), 835-870, 2000.

[10] N. Ben Abdallah, M. Escobedo and S. Mischler, Convergence to the equilibrium for the Pauli equation without detailed balance condition, C.R. Math. Acad. Sci. Paris, 341(1), 5-10, 2005.

[11] N. Ben Abdallah and M.L. Tayeb, Diffusion approximation for the one dimensional BoltzmannPoisson system, Discrete and Continuous of Dynamical Systems-Series B, 4(4), 1129-1142, 2004. 
[12] S. Benachour, Analyticité des solutions des équations de Vlasov-Poisson, Ann. Scuola Norm. Sup Pisa Cl. Sci., 4(16), 1, 83-104, 1989.

[13] F. Berthelin, N Mauser and F. Poupaud, High field limit from a kinetic equation to multidimensional scalar conservation laws, J. Hyperbolic Diff. Eqs., 4(1), 123-145, 2007.

[14] F. Bouchut, Existence and uniqueness of a global smooth solution for the Vlasov-PoissonFokker-Planck system in three dimensions, J. Funct. Anal., 111(1), 239-258, 1993.

[15] F. Bouchut, Renormalized solutions for the Vlasov equations with coefficients of bounded variations, Arch. Ration. Mech. anal., 157, 75-90, 2001.

[16] J. Carrillo, A Jungel, P. Markowich, G. Toscani and A. Unterreiter, Entropy dissipation methods for degenerate parabolic problems and generalized Sobolev inequalities, Monatsh. Math., 133, 1-82, 2001.

[17] S. Chandrasekhar, Radiative Transfer, Dover publications, new York, 1960.

[18] P.H. Chavanis, Generalized thermodynamics and Fokker-Planck equations: applications to stellar dynamics and two-dimensional turbulence, Physical Review E, 68, 36108-36128, 2003.

[19] P. Degond and A. Jungel, High-field approximations of the energy-transport model for semiconductors with non-parabolic band structure, ZAMP, 52, 1053-1070, 2001.

[20] P. Degond and S. Schmeiser, Macroscopic models for semiconductor heterostructures, J. Math. Phys., 39(9), 4634-4663, 1998.

[21] C. De Lellis, Ordinary differential equations with rough coefficients and the renormalization theorem of Ambrosio, Séminaire BOURBAKI, 972, 2006-2007.

[22] R.J. Diperna and P.L. Lions, On the Cauchy problem for the Boltzmann equation; global existence and weak stability, Ann. Math., 130, 321-366, 1989.

[23] R.J. Diperna and P.L. Lions, Ordinary differential equations, transport theory and sobolev spaces, Invent. Math., 98, 707-741, 1989.

[24] R.J. Diperna, P.L. Lions and Y. Meyer, $L^{p}$ regularity of velocity averages, Ann. Inst. H. Poincarré Annal. Non linéaire, 8, 271-281, 1991.

[25] R. Devore and G. Petrova, The averaging lemma, J. AMS., 14(2), 279-296, 2000.

[26] J. Dolbeault, P.A. Markowich, D. Olz and C. Schmeiser, Nonlinear diffusion as limit of kinetic equations with relaxation collision kernels, Archive Rat. Mech. Anal., 186, 133-158, 2007.

[27] J. Dolbeault and C. Schmeiser, The two dimensional Keller segel model after blow-up, DCDS-A, 25, 109-121, 2009.

[28] J. Dolbeault, C. Mouhot and C. Schmeiser, Hypercoercivity for kinetic equations with linear relaxation terms, C.R. Acad. Sci. Paris, 347, 511-516, 2009.

[29] H. Gajewski, On uniqueness of solutions of the Drift-Diffusion-Model of semiconductors devices, Math. Models Methods Appl. Sci., 4, 121-139, 1994.

[30] P. Gérard and F. Golse, Averaging regularity results for PDEs under transversality assumptions, Commun. Pure Appl. Math., 45, 1-26, 1992.

[31] F. Golse and L. Saint Raymond, Velocity averaging in $L^{1}$ for transport equation, C.R. Acad. Sci. Paris, Ser. I, 334, 557-562, 2002.

[32] F. Golse and F. Poupaud, Limite fluide des équation de Boltzmann des semiconducteurs pour une statistique de Fermi-Dirac, Asympt. Analysis, 6, 135-169, 1992.

[33] T. Goudon, Hydrodynamic limit for the Vlasov-Poisson-Fokker-Planck system: analysis of two dimensional case, Math. Models Methods Appl. Sci., 15, 737-752, 2005.

[34] T. Goudon and A. Mellet, On fluid limit for the semiconductors Boltzmann equation, J. Diff. Eqs., 189(1), 17-45, 2003.

[35] T. Goudon, J. Nieto, F. Poupaud and J. Soler, Multidimensional high-field limit of the electrostatic Vlasov-Poisson-Fokker-Planck system, J. Diff. Eqs., 213, 418-442, 2005.

[36] T. Goudon and F. Poupaud, Approximation by homogeneization and diffusion of kinetic equations, Commun. Part. Diff. Eqs., 26, 537-569, 2001.

[37] H. Grad, Asymptotic theory of the Boltzmann equation, Phys. Fluids, 6, 147-181, 1963.

[38] H. Grad, Principles of the kinetic theory of gases, Hand. Phys., 12, 205-294, 1958.

[39] J Haskovek, C. Schmeiser and M.L. Tayeb, Existence and long time behavior of weak solution for the Spherical Harmonics Expansion (or SHE) model coupled to Poisson, in progress.

[40] E. Herst and R. Hunze, Weak solutions of the initial value problem of the unmodified nonlinear Vlasov equation, Math. Meth. Appl. Sci., 6, 262-279, 1984.

[41] C. Kittel, Introduction to Solid State Physics, John Wiley \& Sons, Inc., 1976.

[42] C. Le Bris and P.L. Lions, Renormalized solutions for some transport equations with partially $W^{1,1}$ velocities and applications, Ann. Mat. Pura. Appl., 4(184), 97-130, 2004.

[43] P.L. Lions, Régularity optimale des moyennes en vitesse, C.R. Acad. Sci. Paris, Ser. I, 320, 911-915, 1995.

[44] P.L. Lions and B. Perthame, Propagation of moments and regularity for the Vlasov-Poisson system, Invent. Math., 105, 415-430, 1991. 
[45] P.A. Markowich, F. Poupaud and C. Schmeiser, Diffusion approximation of nonlinear electron phonon collision mechanisms, Modél. Math. Anal. Num., 29(7), 857-869, 1995.

[46] P.A. Markowich, C. Ringhofer and C. Schmeiser, Semiconductor Equations, Springer-Verlag, New York, 1989.

[47] N. Masmoudi and M.L. Tayeb, Diffusion limit of a semiconductor Boltzmann-Poisson system, SIAM J. Math. Anal., 38, 1788-1807, 2007.

[48] N. Masmoudi and M.L. Tayeb, On the Diffusion limit of a semiconductor Boltzmann-Poisson system without micro-reversible process, Commun. Part. Diff. Eqs., 35(7), 1163-1175, 2010.

[49] S. Mischler, On the trace problem for solutions of the Vlasov equation, Commun. Part. Diff. Eqs., 25(7-8), 1415-1443, 2000.

[50] S. Mischler, On the initial-boundary value problem or the Vlasov-Poisson-Boltzmann system, Commun. Math. Phys., 210(2), 447-466, 2000.

[51] J.D. Murray, Mathematical Biology I, An Introduction, Springer, 2002.

[52] F.J. Mustieles, Global existence of weak solutions for a system of nonlinear Boltzmann equattions in semiconductors physics, Math. Meth. Appl. Sci., 14(2), 139-153, 1991.

[53] J. Nieto, F Poupaud and J. Soler, High-field limit for the Vlasov-Poisson-Fokker-Planck system, Arch. Rational Mech. Anal., 158(1), 29-59, 2001.

[54] F. Poupaud, On a system of nonlinear Boltzmann equations of semiconductor physics, SIAM J. Appl. Math., 50(6), 1593-1606, 1990.

[55] F. Poupaud, Diffusion approximation of the linear semiconductor Boltzmann equation: analysis of boundary layers, Asymptotic Analysis, 4, 293-317, 1991.

[56] F. Poupaud, Runaway phenomena and fluid approximation under high fields in semiconductor kinetic theory, Z. Angew. Math. Mech., 72(8), 359-372, 1992.

[57] Yu. P. RAIZER, Gas Discharge Physics, Springer, Berlin, 1997.

[58] L. Reggiani (ed.), Hot Electron Transport in Semiconductors, Springer-Verlag, Berlin, 1985.

[59] G. Rein, Nonlinear stability for the Vlasov-Poisson- the energy-Casimir method, Math. Models Methods Appl. Sci., 10(7), 1027-1045, 2000.

[60] J. Weckler, On the initial-boundary-value problem for the Vlasov-Poisson system: Existence of weak solutions and stability, Arch. Rational Mech. Anal., 130, 145-161, 1995.

[61] C. Ringhofer, C. Schmeiser and A. Zwirchmayr, Moments methods for the semiconductor Boltzmann equation on bounded domains, SIAM J. Math. Anal., 39(3), 1078-1095, 2001.

[62] J. Schaeffer, Global existence of smooth solutions to the Vlasov-Poisson system in three dimensions, Commun. Part. Diff. Eqs., 16(8-9), 1313-1335, 1991.

[63] C. Schmeiser, Macroscopic models for almost elastic nonlinear electron-phonon interaction in semiconductors, SIAM J. Appl. Math., 60(1), 109-120, 2000.

[64] W. Shockley, Problems related to $p-n$ Junctions in Silicon, Solid state Electr, 2, 35-67, 1961.

[65] C. Schmeiser and A. Zwirchmayr, Elastic and drift-diffusion limits of electron-phonon interaction in semiconductors, Math. Models and Meth. appl. sci., 8, 37-53, 1998.

[66] W. Shockley and W.T. Read, Statistics of recombinations of Holes and Electrons, Physical Review, 87, 835-842, 1952.

[67] S.M. SZE, Physics of Semiconductor Devices, second edition, John Wiley \& Sons, New York, 1981.

[68] D. Venturan A. Gnudi, G. Baccarani and F. Odeh, Multidimensional spherical harmonics expansion of Boltzmann equation for transport in semiconductors, Appl. Math. Lett., 5, 85-90, 1992.

[69] J.M. Ziman, Electrons and Phonons: the Theory of Transport Phenomena in Solids, Oxford Press, 1958. 